\title{
Sachregister zu Band 71
}

Die fett gedruckten Zahlen bezeichnen Eigenarbeiten. Bb. = Buchbesprechung.

Abduktion, Fehlen der - oder anormales angeborenes Schielen

131 Ablatio retinae 129,179

nach Unfall 194

Goninsche Operation bei - 97, 119, 200, 385

Addisonsche Krankheit, Pigmentation der Conj unctiva bei - 254

Ähnlichkeitsdiagnose der Au-genmerkmale bei eineiigen Zwil-lingen 35

Akkommodation, Apparat zur Untersuchung der - 384

Akkommodationsparese nach Schädelverletzung 176

Ak kommodationsstörung,Kom-bination der Konvergenzstörung und der - nach Enzephalitis 141

Alkoholinjektionen zur Behand-lung des Blepharospasmus und des spastischen Entropium 135, 169

Amblyopie, das Sehen in $\mathrm{p}^{3} / 41$ len von Schiel-A. 360

Angiomatosis retinae no

Aniridia congenita totalis bi-lateralis 383

Arteriosklerose, Pathologie der - 209

- $\quad$ Entstehung der - bei Jugend-lichen 209

Arzttum in Not 131

Atlas, stereoskopischer - der äu-

Beren Erkrankungen des Auges

133 (Bb.) Atropineinspritzungen, subkon-

junktivale - 129 Augapfel s. Bulbus Augenbewegungen, Störungen

der - bei Kleinhirnbrücken-

winkeltumor 208 Augendruck s. Druck Augenlider s. Li der Augenmuskeln, chronische Myositis der äußeren - 361 Augenmuskellähmungen, teil-

mattierte Gläser bei - 257 A ugenmuskelstörungen 190

- $\quad$ bei Myasthenie 292Augentuberkulose, typischer und

atypischer Verlauf bei - 374 Azetylcholin 129

B

Behrsches Syndrom, linksseiti-

ges 379 Belastungsproben bei Glaukom

101 Bindehaut s. Conjunctiva Blepharitis, Röntgenbehandlung

der - 202 Blepharochalasis 182 Blepharospasmus, Alkoholinjektionen zur Behandlung des -

135, 169 Blutdruck, Einfluß des - auf den

intraokularen Druck 259 Blutzucker, Augenerscheinungen

bei erheblichen Schwankungen

des - 197 Brechungsfehler, Ausgleich sämt-

licher - durch geschliffene Haft- 
gläser 128 Buckel, episkleritischer 115 Bulbus, das -bild der Lidspalte

176 Bulbusvolumen, Apparat zur Be-

stimmung des -182

$\mathrm{C}$

Carcinoma metastaticum der Chorioidea bei normaler Seh-schärfe 204

Chorioidea, metastatisches Kar-zinom der - an einem Auge mit normaler Sehschärfe 204

Chorioideasarkome, Prognoseder

- $\quad 387$

Chorioiditis e lue congenita 109

- tuberculosa, zentrale noConjunctiva, Pigmentation der --

bei Addisonscher Krankheit 254

Zytologie der - bei Trachom 312

luetische Erkrankung der - 205

syphilitische Berufsinfektion der

- bei einem Arzt 210Contusio bulbo, seltener Augen-

hintergrund nach - 203 Cornea, Regeneration großer Epi-theldefekte der -- in

perforierende Verletzung der--durch einen Steinsplitter 160

- Randatrophie 108

-Randektasie 108

Corpus ciliare, traumatische Epi-thelimplantationszyste des - 211

Sachregister zu Band 71.

393

Corpus ciliare, Sarkom des -

383 Cyclitis bei Katarakta electrica 156

$\mathrm{D}$

Descemeti - Pigmentring bei

Pseudosklerose 108 Diphtherieinfektion am Auge

und ihre Beeinflussung durch

Heilserum 354 Dispensärarbeit gegen Trachom

in Kiew 383 Druck, intraokularer, Einfluß

der Plasmavermehrung auf den

$-\quad 258$

- $\quad$ Einfluß des Blutdruckes auf den

$-\quad 259$

- $\quad$ Verhalten des - nach Vorder-kammerpunktion bei Glaukom 48

E Eisensplitterverletzung no Endokrine Augenstörungen353 Endokrine Störungen, Beziehungen der Gefäßhaut zu -r 353 Endothelioma palpebrae 347 Entropium, Alkoholinjektion zur Behandlung des spastischen -

135 Enukleation, Prognose der - bei

Melanosarkom der Uvea 178 Enzephalitis, Symptomatik der

subakuten - 117 - Kombination der Akkommoda-

tions- und Konvergenzstörung

nach - 141 Epithelimplantationszyste,

traumatische - des Ziliarkörpers

211 Episkleritischer Buckel 115 Exophthalmus, pulsierender 108 
intermittierender 207, 247, 383

Sympathikusparese bei -183

$\mathrm{F}$

Farbenblindheit, Filtergebrauch bei - 127

Fernrohrlupe, Lesepult für - 174

Feuerarbeiter, vorzeitige Presby-opie der - 211

Fieberbehandlung der tabischen Sehnervenatrophie 191

Filtergebrauch bei Farbenblindheit 127

Frauenhaar als Nahtmaterial 201

Fremdkörper, röntgenologische Lagebestimmung intraorbitaler - durch Einspritzen von Jodipin und Lufteinblasung in den Tenon-schen Raum 190

Frühplastik, Indikation der - nach Verätzung des Auges 211

Führungsbewegungen, Patholo-

gie der - 208 Fusionszwang, Einfluß des - auf

das dissoziierte Schielen 184

G

Galamerzsche Operation bei pannösem Trachom 384

Gefäßhaut, Beziehungen der - zu endokrinen Störungen 355

Gesichtsfeld für Licht und der Pupillenreflex 130

Gesichtsfeldbestimmung am Bjerrumschirm 354

Gesichtsfeldzentrum, Tafeln zur binokularen Untersuchung des - mit dem Stereoskop 212 (Bb.)

Glasmacherstar, Schwierigkeiten in der Diagnose und in der Be-urteilung der Dauerinvalidität

bei -112

Glaukom 104

juvenile inversum 171

Belastungsproben bei - 101

Verhalten des intraokularen Druckes nach Vorderkammer-punktion bei - 48

Grundlagen der physikalisch-diä-tetischen Behandlung des - 82:

Goninsche Operation 97, 119, 200, 385

- $\quad$ Technik der -363

- Zielophthalmoskop für die - 307Grenzstrahlen, Behandlung der

Erkrankungen des vorderen Bul-busabschnittes mit - 1, 101

$\mathrm{H}$

i Haemangioma conjunctivae zu-gleich mit Naevus vasculosus der Schläfe 183 Haftgläser,

Ausgleich sämtlicher Brechungsfehler durch geschlif-fene - 128

- $\quad$ Verwendbarkeit der - bei durch-bohrenden Wunden des Auges 168

Handbuch, Kurzes der Ophthal-

mologie, Bb. 388 Heterochromia iridis 384

$-$

H. beim Ka-

und Iridozyklitis 205Hornhaut s. CorneaHypophysentumoren 185 Hypotonie, Prellungs-

ninchen 198

I

Ignipunktur, Goninsche bei Netz-hautablösung 97, 119, 200, 3 ?5

Iridozyklitis und Heterochromia iridis 205

Iris, Heterochromie der - 205, 384

Iriszyste, posttraumatisch entstan-dene seröse -174 
394

Sachregister zu Band 71.

Iritis, langjährige rezidivierende -1/8 von den Gaumentonsillen aus-gehend 107

- $\quad$ und Menstruation 386

K

Kalilaugenverätzung des Ge-sichts und beider Augen 109

Katarakt, eine eigenartige - beim Kaninchen 114

bei myotonischer Dystrophie 106

Trockensubstanzbestimmungen bei menschlicher -373

- $\quad$ electrica cum cyclitide 156Kataraktextraktion, Beschrei-

bung der - Jung-Stillings und

seines Instrumentariums 198 Kayser - Fleischerscher Ring,

Grundlagen des - bei Wilson-

scher Krankheit 186 Keilbeinmetastase eines Ovarial-

sarkoms mit totaler Ophthalmo-

plegie 183 Keratitis parenchymatosa 109 Keratokonus 175

- 6 Jahre getragene Kontaktgläserbei - 95

Kleinhirn - Brückenwinkeltu-mor, Störungen der Augenbe-wegungen bei - 208

Kontaktgläser, 6 Jahre getragene

- $\quad$ bei Keratokonus 95Kontaktschalen 205Konvergenz, ein Faktor der -

359 Konvergenzstörung, Kombina-

tion der Akkommodationsstö-

rung und der - nach Enze-

phalitis 141 Konvergenzzentrum, physio-

logische Beziehung zwischen dem

Seitenwenderzentrum des Auges

und dem - 160 Krankenschein gefällig ? 131 Krönleinsche Operation eines

Optikustumors 203

L

Lesepult für Fernrohrlupe 174 Leuchtende Strahlen, schwere

Blendung durch - 211 Licht, neutrales 353 Li der, Erkrankung der - nach

Teakholzverarbeitung 211

Endotheliom der - 347

Behandlung der Karzinome der

- mit Röntgenstrahlen 124Lidspalte, das Augapfelbild der-

176 Lidzerreißung durch Wasserstrahl 204

Linse, Extraktion einer subluxier-ten getrübten - in der Kapsel 171

Lues, luetische Erkrankung der Bindehaut 205

Lymphom der Conjunctiva 198

$\mathrm{M}$

Maculae corneae, skrofulöse 108 Macula lutea, kortikale Lage der

$-\quad 115$

Heterotopie der - 189

indirekte traumatische Ruptur der - 211

Magnetoperation, diasklerale 107 Malaria, Augenveränderungen bei 
$-\quad$ in

Melanosarkom der Uvea, Prognose

der Enukleation bei - 178 Melanosis bulbi 204 Menstruation und Iritis 386 Mikrophthalmus 112

Milchpräparat, ein neues - zur

unspezifischenReizkörpertherapie

381 Myasthenie, Augenmuskelstörun-

gen bei -292

- $\quad$ mit Thymusgeschwulst, Augen-symptome bei einem Fall von -187

Myositis, chronische - der äuße-

ren Augenmuskeln 361 Myotonie bei Auswärtsschielen 107 Myotonische Dystrophie, Katarakt

bei -106

$\mathrm{N}$

Naevus vasculosus der Schläfe zugleich mit Haemangioma con-junctivae 183

Nahepunktbestimmung, Tech-nik der - 152

Nervus facialis, Einfluß der Läh-mung des - auf die Tränen-absonderung und die Funktion des tränenableitenden Apparates 201

Nervus oculomotorius, rezidivierende Parese des - und Heredi-tät 197

Nervus opticus, Veränderungen am - 193

- -atrophie, kavernöse 271

-atrophie bei Turmschädel 109

-atrophie, Bedeutung d. Röntgen-aufnahme des Schädels für die Diagnose der pseudoglaukomatösen -124

-atrophie, Behandlung der tabi-schen - 12

-atrophie, Fieberbehandlung der tabischen 191

Sachregister zu Band 71.

395

Nervus opticus-Tumor durch

Krönleinsche Operation entfernt

203 Netzhaut s. Retina Netzhautablösung s. Ablatio

retinae Neurofibrome, zwei - in einem

Auge 272 Neutrales Licht 353 Nystagmus, willkürlicher - 195

periodisch umschlagender - 181

der Bergleute 259

- als Gegenstand der Gesetz-gebung 226

0 Ophthalmologie, Kurzes Hand-

buch der-Bb. $388 \mathrm{O} p \mathrm{~h} \mathrm{th}$ a $1 \mathrm{~m}$ o p $1 \mathrm{e}$ g i e, totale - bei

Keilbeinmetastase eines Ovarial-

sarkoms 183 Optochin-Iontophorese, Be-

handlung des Ulcus corneae ser-

pens mit -125

$P$

Pemphigus der Lider und Binde-

häute 102 Perimetrie 382 Phthisisbulbi, Knochengebilde im

Auge bei - 115 Plasmavermehrung, Einfluß der

- $\quad$ auf den normalen Augendruck258 
Plasmoma conjunctivae 172 Prellungshypotonie beim Ka-

ninchen 198 Presbyopie, vorzeitige - der

Feuerarbeiter 211 Pseudo-Basedow, enzephaliti-

scher - 118 Pseud osklerose, Descemeti-Pig-

mentring bei - 108 Pterygium, Refraktion bei - 237 Punktlichtlampe als Lichtquelle

für Augenspiegeluntersuchung 174 Pupillomotorische Mittel, Wir-

kungsweise der - bei örtlich

umschriebener Applikation 386 Pupillenreflex und das Gesichts-

feld für Licht 130

E

Refraktion bei Pterygium 237 Reizkörpertherapie, ein neues Milchpräparat zur unspezifischen

- 381

- Wirkung der - auf das Auge 196 Retinablutungen, klinische Ver-

wertung der - 102 Retinariß ohne Abhebung 96 Rindennahtpunktierung, atypi-

sche 95

Röntgenauf nahme des Schädels, Bedeutungder -für die Diagnose der pseudoglaukomatösen

Seh-nervenatrophie 124

Röntgenbehandlung der Blepharitis 202

- der Lidkarzinome 124Röntgenographische Lagebe-

stimmung intraorbitaler Fremd-körper durch Einspritzen von Jodipin und Lufteinblasung in den

Tenonschen Raum 190 Roßhaar als Nahtmaterial 201 Rücklagerung, verstärkte - bei

Auswärtsschielen 107

S Salbenspritze 96 Salvarsan, histologischer Nachweis

des - im Auge 114 Sarkom der Uvea 203 Schädelverletzung, Akkommo-

dationsparese nach - 176 Schanker der Lidbindehaut bei

einem Arzt durch Berufsinfek-

tion 210 Schattenprobe, eine einfache Er-

klärung der - 106 Scheinschielen und Schielen in

der Kunst 188 Schielen, angeborenes anomales -

oder Fehlen der Abduktion 131

das Sehen in Fallen von Schiel-amblyopie 360

Einfluß des Fusionszwanges auf das dissoziierte - 184

Scheinschielen und - in der Kunst 188

ein Faktor des Strabismus con-vergens 359

verstärkte Rücklagerung bei Strabismus divergens 107

Schwefelgrubenarbeiter, Augen-schädigungen bei - 128

Sehnerv s. Nervus opticus

Sehschärfe, Tafeln zur Unter-suchung der ;- 384

Sehschulen 114

Seitenwenderzentrumdes Auges, physiologische Beziehung zwi-schen dem Konvergenzzentrum und dem -160

Sella turcica, Veränderungen der

- 193 Simulation, Röhren von Gratama

zur Aufdeckung von - 115 Skleritis, ringförmige - mit Vor-

treibung der Hornhaut 102 Sklera, blaue, ein Stammbaum

der - und ihre vererbungs- 
theoretischen Untersuchungen 328

396

Sachregister zu Band 71.

Skopolamin-Ephetonin -Euko-

dal-D ämmerschlaf 177 Skotometer, ein neues - 204 Spiegelbildsymmetrie der Au-

genmerkmaie bei eineiigen Zwil-

lingen 35 Sprengkapselverletzung, per-

forierende - beider Augen 109 Stauungspapille im experimen-

tum crucis hominis 275 Stereoskopischer Atlas der äu-

Beren Erkrankungen des Auges

133 (Bb.) Strabismus s. Schielen Sympathikusheterochromie,

experimentelle - 235 Sympathikusparese bei Exoph-

thalmus pulsans 183

$\mathrm{T}$

Tafeln zur binokularen Untersu-

chung des Gesichtsfeldzentrums

mit dem Stereoskop 212 (Bb.) Tabischer Sehnervenschwund,

Behandlung des - 12 - Fieberbehandlung des - 191 Teakholzverarbeitung, Lid-

erkrankung nach - 211 Teilmattierte Gläser bei Augen-

muskellähmungen 257 Thymusgeschwulst mitMyasthe-

nie, Augensymptome bei einem

Fall von - 187 Tonsillen, langjährige rezidivie-

rende Iritis, von den - aus-

gehend 107 Trachom 383, 384, 385

Frequenz des - in Ostpreußen 217

und Konstitution 206

Zytologie der Bindehaut bei -312

- Bekämpfung des - 213 Trakolysin 385 Tränenabsonderung, Einfluß der

Fazialislähmung auf die - 201 Tränenalkalose bei Trachom 385 Tränendrüse, Rezidiv eines vor

drei Jahren operierten Tumors

der - 109 Tränensackerkrankung, radi-

umbestrahlte tuberkulöse 95 Trichloräthvlen, Giftwirkung des

- auf das Auge 211

Trockensubstanzbestimmun-gen bei menschlichen Staren 373

Tuberkulöse, Bedeutung gestei-gerter Vitaminzufuhr für die Behandlung der - 387

Turmschädel mit Optikusatrophie 109

$\ddot{\mathrm{u}}$

Überempfindlichkeiten in der Ophthalmotherapie 180, 283

Ulcus corneae serpens, Behandlung des - mit Ôptochin-Ionto-phorese 125

- Behandlung des - mit Winter-grünöl 176

Ultraviolette Strahlen, schwere Blendung durch - 211

Unfall, Netzhautablösung nach - 194

Unfallkunde 131, 211

V Verätzung des Auges, Indikation

der Frühplastik nach - 211 Versicherungskunde 131, 211 Vitaminzufuhr, Bedeutung ge- 
steigerter - für die Tuberkulose-

therapie 387 Vorderkammerpunktion bei

Glaukom, Verhalten des intra-

okularen Druckes nach - 48

W

Wilsonsche Krankheit, Grund-

lagen des Kayser-Fleischerschen

Ringes bei der - 186 Wintergrünöl, Behandlung des

Ulcus serpens mit --176 Wirtschaftsfragen des Ruhrberg-

baus 132

$\mathrm{Z}$

Zielophthalmoskop für die operative Behandlung der Netz-hautabhebung nach Gonin 307

Ziliargefäße 258

Z. Zonulalamelle und ihre Ablösung von der Linse 258

Zwillinge, Ähnlichkeitsdiagnose der Augenmerkmale und Spiegelbildsymmetrie bei eineiigen 35 